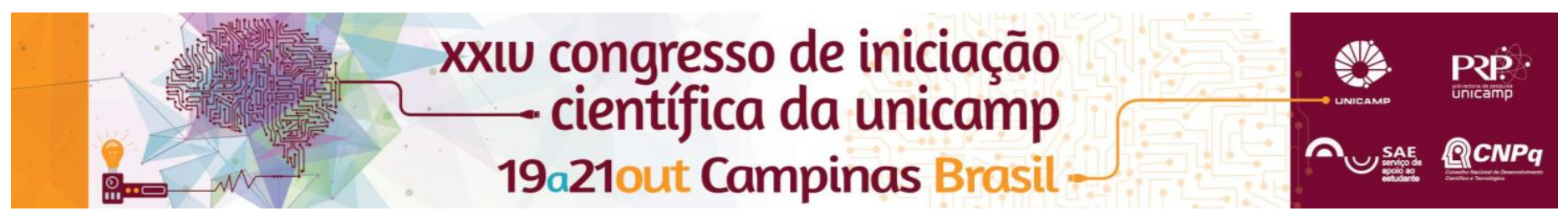

\title{
Stability of lutein delivery nanoemulsions during storage
}

\author{
Daniela Z. Demasi (IC) *, Luiza C. O. Carvalho (IC), Daniele B. Rodrigues (PG) e Lilian R. B. Mariutti (PQ).
}

\begin{abstract}
The daily intake of lutein can lead to the accumulation of this carotenoid in the retina, helping to protect the eyes against age-related macular degeneration. To increase the consuption of lutein by producing fortified food products, a technical difficult is faced due to the difficult to incorporate lipophilic compounds in water based matrices. In this work, we developed an oil-in-water nanoemulsion for lutein delivery and evaluated its chemical and physical stability during a four week refrigerated storage $\left(1^{\circ} \mathrm{C}\right)$ in the dark and an accelerated storage experiment $\left(60^{\circ} \mathrm{C}\right.$ for 12 days).
\end{abstract}

\section{Key words:}

Emulsion, carotenoids, stability

\section{Introduction}

Age-related macular degeneration (AMD) is a progressive degenerative disease that affects the central portion of the retina (macula), resulting in loss of central vision. ${ }^{1}$ The life expectance of the general population is increasing, so the use of lutein to prevent the development of degenerative diseases becomes an interesting alternative. Oil-in-water nanoemulsions are an option of delivery system to lipophilic compounds because they allow the incorporation of these compounds to aqueous matrices without significant increases it the total lipid content. We developed an oil-in-water nanoemulsion for lutein delivery without added antioxidants and evaluated and its chemical and physical stability in two storage situations: during a four week refrigerated storage $\left(1^{\circ} \mathrm{C}\right)$ in the dark and an accelerated storage experiment $\left(60^{\circ} \mathrm{C}\right.$ for 12 days).

\section{Results and Discussion}

The lutein rich oil used in the nanoemulsion formulation was obtained by extracting the carotenoids from lyophilized marigold petals directly in soy oil by magnetic stirring at $50^{\circ} \mathrm{C}$, followed by vortexing and overnight magnetic stirring at room temperature under nitrogen atmosphere. Nanoemulsions were prepared by microfluidization (3 cycles, 10000 psi) with three different carotenoid concentrations (4, 12 and $19 \mu \mathrm{g}$ lutein $/ \mathrm{mL}$ ), $5 \%$ soy oil and $1.5 \%$ tween 20 aqueous solution.

In fresh nanoemulsions, the higher the carotenoid content the higher the color parameters $a^{*}$ and $b^{*}$ values due to an increase in orange color.

All the nanoemulsions were highly stable during both storage conditions. No physical separation was observed in any formulation at any time. Particle size $\left(254 \mathrm{~nm} / 60{ }^{\circ} \mathrm{C}\right.$ and $250 \mathrm{~nm} / 1^{\circ} \mathrm{C}$ ) and zeta potential (- $36 \mathrm{mV} / 60^{\circ} \mathrm{C}$ and $\left.30.2 \mathrm{mV} / 1^{\circ} \stackrel{\circ}{ } \mathrm{C}\right)$ values did not change during storage regardless the the carotenoid content of the nanoemulsion. Figure 1 shows that loss of color occurred at the same rate in all the formulations in the accelerated storage experiment. Neither carotenoid degradation nor changes in color parameters were observed during refrigerated storage in all formulations (Figure 1).
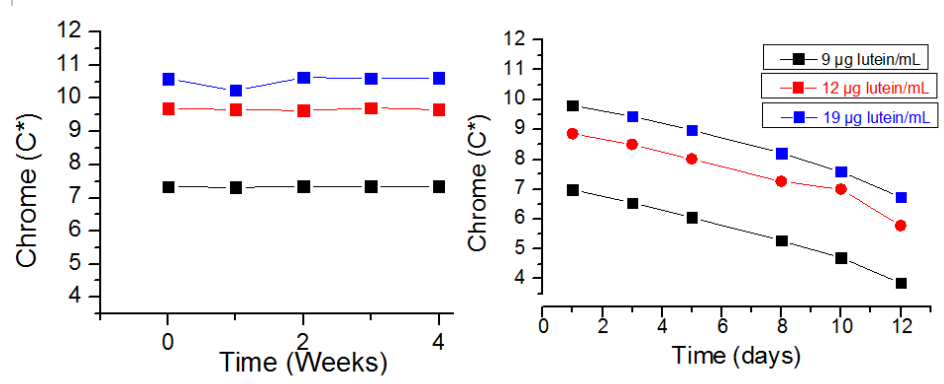

Figure 1. Values of $C^{*}$ (chrome) of emulsions during refrigerated storage (left) and accelerated storage (right). The values were calculated using equation 1.

$$
C^{*}=\sqrt{\left(a^{*}\right)^{2}+\left(b^{*}\right)^{2}}
$$

\section{Conclusions}

All nanoemulsions were stable during storage. These stable nanoemulsions showed to be a good option for lutein delivery because they allow the incorporation of this compound to aqueous matrices without significant increases in the total lipid contents. Nanoemulsions stored in $60^{\circ} \mathrm{C}$ lost the orange color within 15 days due to carotenoid degradation. Although the physical stability of the nanoemulsions was maintained during the accelerated storage experiment, it will be necessary to add antioxidants to increase lutein thermostability.

\section{Acknowledgement}

CNPq (Projeto Universal-455748/2014-4), Bolsa IC Fapesp (D.Z.D., Proc. 2014/27302-0), Projeto EMU Fapesp (Proc. 2009/54137-1) e Bolsa SAE (L.C.O.C., $\mathrm{PIBIC)}$.

${ }^{1}$ Seddon, J.M., Ajani, U.A., Sperduto, R.D. (1994) Dietary carotenoids, vitamins A, $\mathrm{C}$, and $\mathrm{E}$, and advanced age-related macular degeneration. Eye Disease CaseControl Study Group. JAMA, 272, 1994, 1413 - 1320. 